\title{
Silver coordination polymers with isonicotinic acid derived short polyethylene glycol - Synthesis, structures, anion effect and solution behavior
}

\author{
Katharina M. Fromm*, Jorge L. Sagué, Adeline Y. Robin \\ University of Fribourg, Department of Chemistry, Chemin du Musée 9, CH-1700 Fribourg, Switzerland
}

\begin{abstract}
Silver coordination compounds and one-dimensional polymers have been studied, using a linear, flexible $\mathrm{N}$-donor ligand. The solid state structures are well known and show panoply of different structures including a large number of polymorphism, pseudo-polymorphism and isomers. We have studied these structures under the influence of anions, solvents and crystallization conditions, studying also some solution effects. We here present an overview with highlights of some case studies of the synthesis, structures, anion and solvent effects as well as solution behavior during formation of silver coordination polymers. Finally, we will give an outlook on the potential applications of these materials.
\end{abstract}

\section{Introduction}

Silver coordination polymers are studied by many groups due to their structural diversity $[1,2]$ as well as potential use e.g. in catalysis [3], in gas sorption [4], luminescence [5] or as antimicrobial coating [6,7]. In most cases, the solid state structures are the most prominently discussed results obtained in this kind of research areas, while solution studies of the formation of silver coordination polymers remain rare [8]. Nevertheless, this is an important issue in the understanding of the formation of these structures. Thus, we here would like to present (a) an overview on the different structures as a function of solvent, anion and crystallization conditions, and (b) some case studies taken from our previous research.

We started to investigate silver coordination polymers using the neutral ligand ethanediyl bis(isonicotinate) Li1 (Scheme 1) $[9,10]$ and its derivatives, which can be modified by simple elongation of the middle spacer, using diethylene glycol [11], triethylene glycol $[12,13]$ and tetraethylene glycol $[13,14]$ as starting materials for the synthesis of the ligands. In this manuscript however, we will focus on the shortest variant of the ligand, Li1.

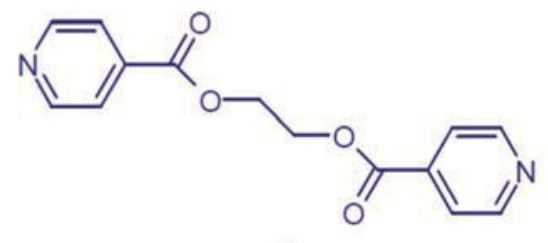

,anti

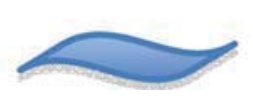

Scheme 1. Ligand Li1 in its two quasi equivalent conformations and their schematic representation.

\section{Results and discussion}

We have shown that the free ligand Li1 crystallizes in the anti conformation, while both conformations, anti and gauche, have a very similar energy and a low energy of activation [13]. Upon coordination of the ligand Li1 to silver ions, we thus expected solid state structures with both ligand conformations. So far, we have obtained the structural motifs described in Scheme 2 [16-18].

In total, seven different coordination polymers were obtained in our group so far with Li1, of which only one has the ligand in the gauche conformation. Three structures feature double chains, two of which contain solvent. The other four compounds follow either type 1 or type 2 structures. All seven structures will be briefly highlighted for their synthesis, solvent and anion effects.

\subsection{Synthesis of silver coordination polymers}

While the ligand Li1 is well soluble in apolar solvents, the silver salts need to be dissolved in polar solvents. Direct mixing of the two solutions leads often immediately to insoluble (crystalline)<smiles>O=C(OCCOC(=O)c1ccncc1)c1ccncc1</smiles>

gauche'

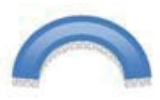

\footnotetext{
* Corresponding author. Tel.: +4126300 8732; fax: +41263009738.

E-mail address: Katharina.fromm@unifr.ch (K.M. Fromm).
} 


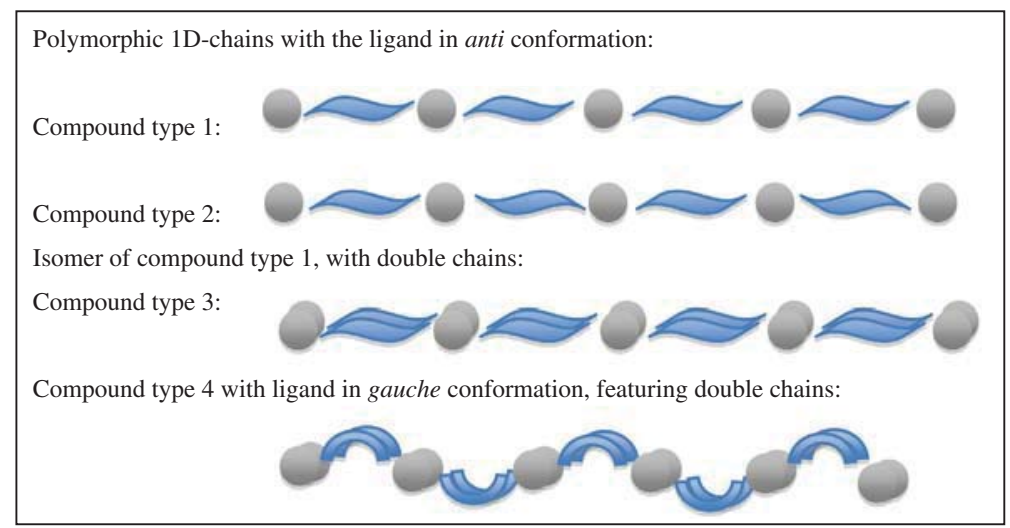

Scheme 2. Different coordination polymer motifs obtained for the reaction of silver salts with Li1 (silver grey, ligand blue).

powders. We therefore slowed down the polymer formation by separating the two reactants in their respective solvents into two arms of an H-tube (Scheme 3). Both compartments are then connected with each other by layering a mixture of both solvents on top such that the horizontal part of the H-tube is filled, or by using a third solvent as connecting solvent. The mixture of solvents or third solvent serves then as buffer for the formation of the polymer. By letting the diffusion occur at room temperature or in the fridge or freezer, single crystals (Picture 4) can grow slowly over several days, weeks or months. Other options to obtain coordination polymers of silver salts with Li1 comprise direct mixing in good solvents for both starting materials, followed by slow evaporation of the solvent mixture or direct layering of the two reactant solutions without buffer as described for the H-tube. In some cases, when the solubility of one or both reactants is low in the chosen solvent, a microwave reaction can also be performed. There are no unique recipes for obtaining single crystals of coordination polymers, and each ligand - metal salt system needs to be finetuned for crystallization conditions.

\subsection{Solvent effect}

A quite large number of our coordination compounds with Li1 was obtained with silver nitrate as starting silver compound. We were thus able to study the solubility effect here in more detail.

(i)

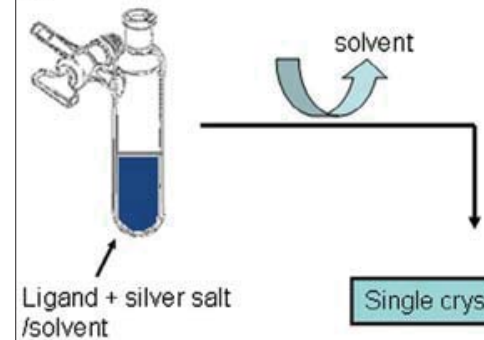

(ii)

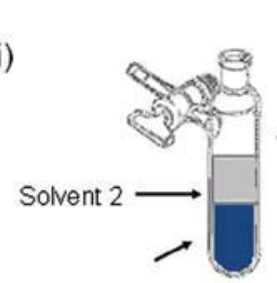

Ligand + silver salt /solvent 1
For the first examples, the ligand Li1 is always dissolved in THF, while the silver nitrate is dissolved in different solvents [16-18].

- Ethanol as solvent for $\mathrm{AgNO}_{3}$ : Among the tested solvents for silver nitrate, ethanol was a less good solvent for the metal salt. The dissociation of silver nitrate in ethanol is apparently incomplete and coordination with Li1 yields thus a solvate of $\mathrm{AgNO}_{3}$ in which 1D-chains of $\mathrm{AgNO}_{3}$ are connected via ligand molecules adopting the anti conformation into a $2 \mathrm{D}$ sheet structure. The ratio $\mathrm{Ag}^{+}: \mathrm{Li} 1$ is $2: 1$ (Fig. 1 ).

- Water as solvent for $\mathrm{AgNO}_{3}$ : Water is the better solvent for the silver salt, and with the ligand dissolved in THF, this reaction yields $1: 1$ compounds with different amounts of co-crystallizing water molecules of composition $\left[\mathrm{Ag}(\mathrm{Li} 1) \mathrm{NO}_{3}\right] \cdot\left(\mathrm{H}_{2} \mathrm{O}\right)_{n}, n=0,1,2$ (Fig. 2). The "dry" compound without solvent is obtained with 1D Ag-Li1-Ag-Li1 chain arrangements of type 2. The monohydrate compound is to date the only silver coordination compound in which the ligand adopts a gauche conformation, giving thus a structure of type 4 . Finally, the dihydrate has a third structure of type 3 [16-18].

The dihydrate compound of structure type 3 can also be obtained if the ligand is dissolved in ethanol instead of THF while the silver nitrate is dissolved in water.

(iii)

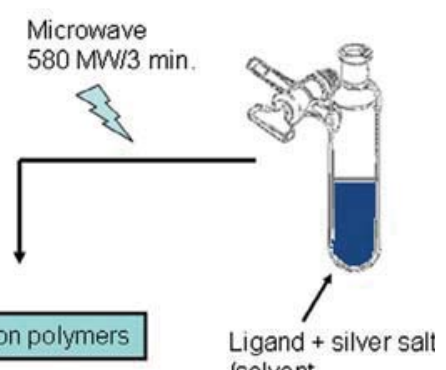

/solvent

Scheme 3. Possible approaches for obtaining single crystals of silver coordination polymers [11]. 


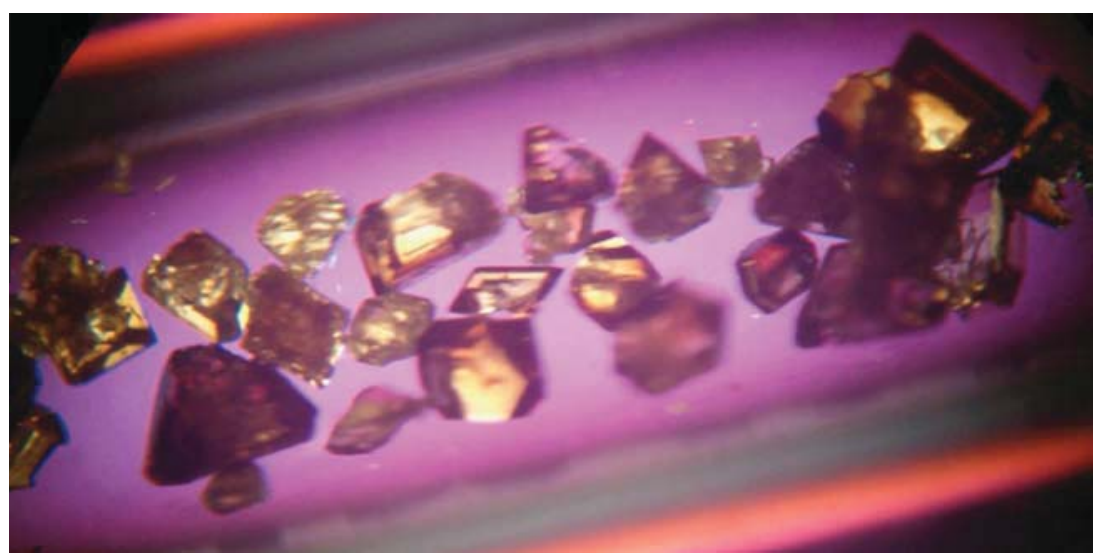

Picture 1. Single crystals of silver coordination polymer [15].

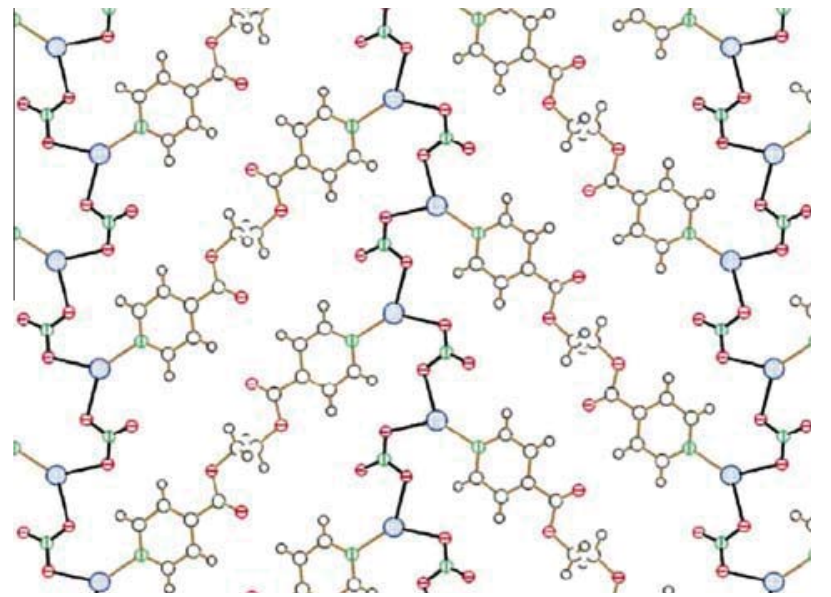

Fig. 1. Excerpt of $\left[\mathrm{AgNO}_{3}(\mathrm{Li} 1)_{0.5}\right]$ (color code: Ag blue, $\mathrm{O}$ red, $\mathrm{N}$ green, $\mathrm{C}$ colorless, $\mathrm{H}$ grey) [16-18].
However, if both the ligand and silver nitrate are dissolved in either ethanol, acetonitrile or in $\mathrm{CH}_{2} \mathrm{Cl}_{2}$, thus both in the same solvent, then always the same 1:1 compound is obtained with structure type 1 (Fig. 3 ). This seems to be the case if slow crystallization via diffusion is tested, but also if the two reaction partners are mixed to yield directly a microcrystalline solid. In the latter cases, the powder X-ray diffractograms are always compared to the ones calculated from single crystal data in order to identify the compound and to ensure its purity.

In all these cases, the nitrate anion is more or less strongly coordinated to the metal ion. We could show, that the $\mathrm{Ag}-\mathrm{O}$ (nitrate) distances in the final structures seems to depend on the solubilizing power of the solvent. This was resumed in the following graphic (Fig. 4). Thus, the lower the solubility of the silver nitrate in the used solvent, the shorter the $\mathrm{Ag}-\mathrm{O}$ distances in the final compounds.
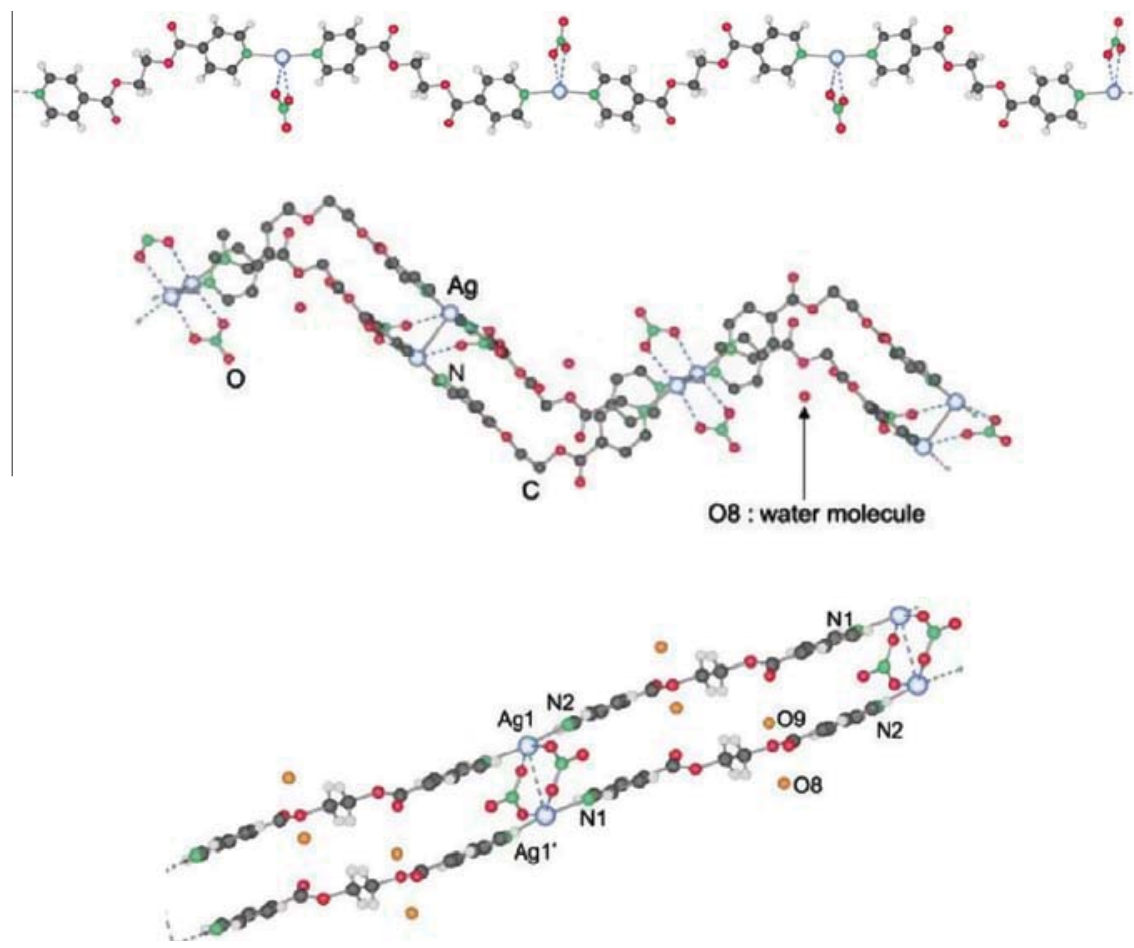

Fig. 2. Excerpts of the solid state structures of $\left[\mathrm{Ag}(\mathrm{Li} 1) \mathrm{NO}_{3}\right] \cdot\left(\mathrm{H}_{2} \mathrm{O}\right)_{n}, n=0$ (top, type 2 ), 1 (middle, type 4 ), 2 (bottom, type 3 ) (color code: Ag blue, $\mathrm{O}$ red or orange (water), $\mathrm{N}$ green, $\mathrm{C}$ grey, $\mathrm{H}$ colorless) [16-18]. 


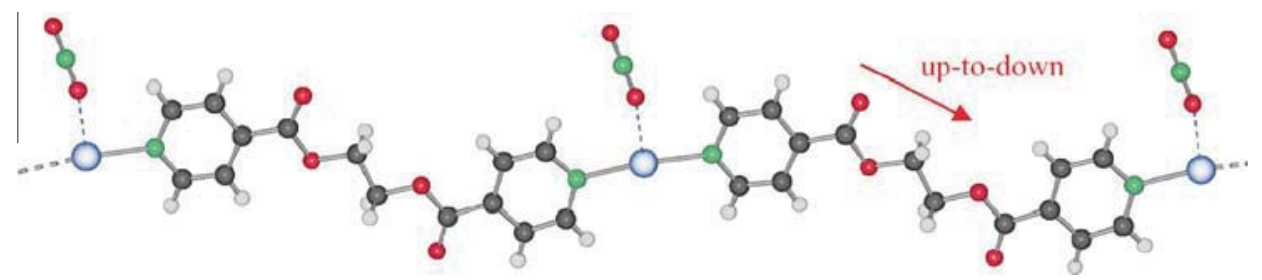

Fig. 3. Structure type 1 realized for $\left[\mathrm{Ag}(\mathrm{Li} 1) \mathrm{NO}_{3}\right]$ (color codes as above) [16-18].

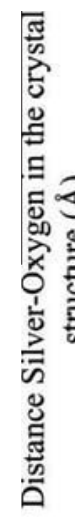

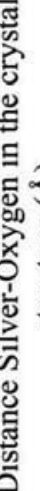

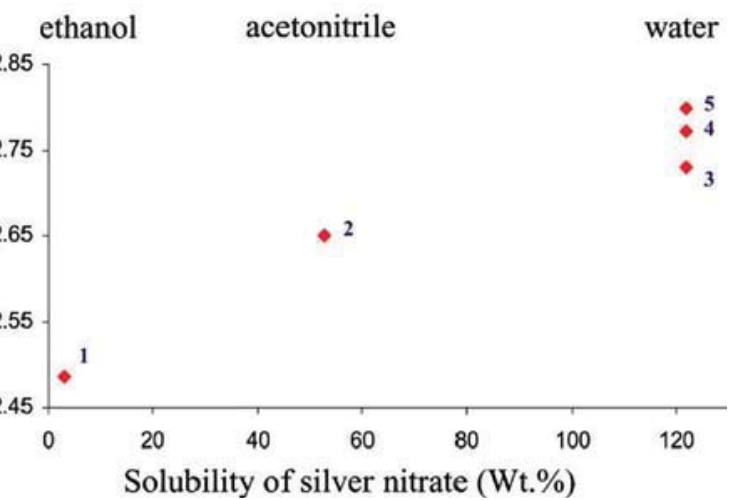

Fig. 4. Graphic showing the Ag-O(nitrate) distances as a function of solvent used for the preparation of the compounds. Compounds 1-5 are, respectively $\left[\mathrm{AgNO}_{3}(-\right.$ Li1 $\left.)_{0.5}\right],\left[\mathrm{Ag}(\mathrm{Li} 1) \mathrm{NO}_{3}\right]$ (type 1 ), and $\left[\mathrm{Ag}(\mathrm{Li} 1) \mathrm{NO}_{3}\right] \cdot\left(\mathrm{H}_{2} \mathrm{O}\right)_{n}, n=0,1,2[13]$.

\subsection{Anion influence}

Nitrate is known to be an anion with multiple coordination possibilities. Indeed, we could show that it can coordinate via one, two or all three oxygen atoms to silver ions in the solid state. If an oxygen atom of nitrate remains free and does not coordinate to the cation, it may undergo $\mathrm{H}$-bonding with solvent molecules, as shown for example for the dihydrate compound with structure type $3[16-18]$.

We therefore exchanged the nitrate anion for the less coordinating hexafluorophosphate $\mathrm{PF}_{6}{ }^{-}$and triflate $\mathrm{CF}_{3} \mathrm{SO}_{3}{ }^{-}[7,13]$. For the former, a structure type 2 is observed, while for triflate, structure type 1 is formed (Fig. 5). Both compounds can be obtained via the diffusion method using an H-tube with THF as solvent for the ligand and water as solvent for the metal salt, while the triflate derivative was also made by microwave synthesis and subsequent slow cooling of the solution.

In contrast to the 2D-structure of the nitrate anion (flat molecule), both the $\mathrm{PF}_{6}{ }^{-}$and the $\mathrm{CF}_{3} \mathrm{SO}_{3}{ }^{-}$anion are three-dimensional
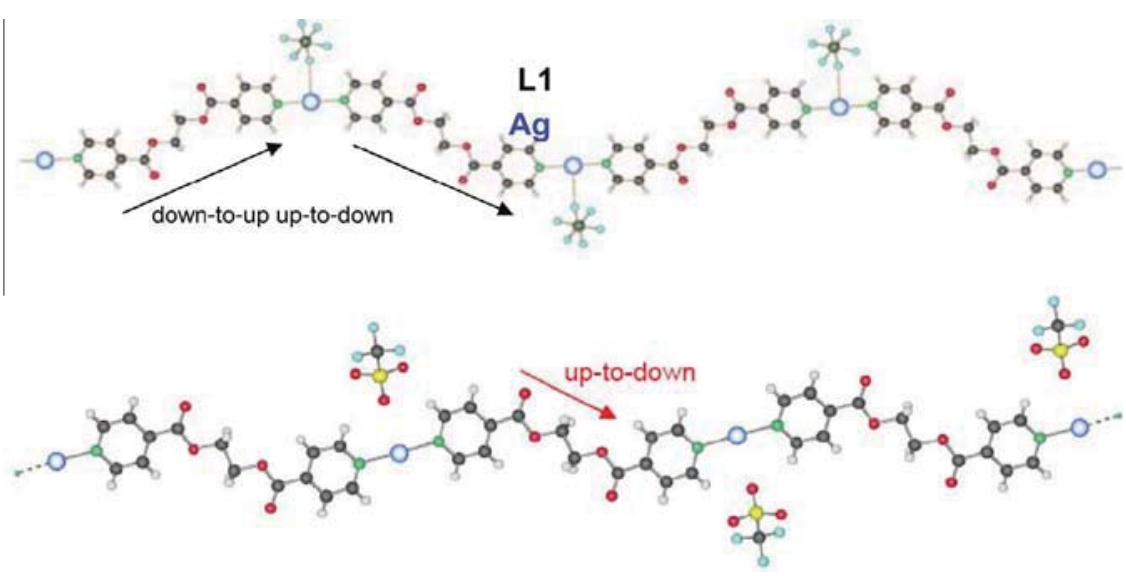

Fig. 5. Excerpts of the solid state structures of $\left[\mathrm{Ag}(\mathrm{Li1}) \mathrm{PF}_{6}\right]$ (top, type 2) and [ $\left.\mathrm{Ag}(\mathrm{Li1}) \mathrm{CF}_{3} \mathrm{SO}_{3}\right]$ (bottom, type 1 ) (color codes as in Fig. 2, $\mathrm{F}$ turquoise, $\mathrm{S}$ yellow, $\mathrm{P}$ dark green) [7].

Table 1

Relative amounts of different complexes in solution, as a function of metal to ligand ratio and time $(0,24 \mathrm{~h})$, of Li1 (abbreviated $\mathrm{L}$ in the table) and $\mathrm{AgNO}{ }_{3}$ in a mixture of acetonitrile: $\mathrm{CH}_{2} \mathrm{Cl}_{2}$ of ratio $10: 1$ [15].

\begin{tabular}{|c|c|c|c|c|c|c|}
\hline & \multicolumn{2}{|l|}{$\mathrm{M}_{2} \mathrm{~L}$} & \multicolumn{2}{|l|}{$\mathrm{ML}$} & \multicolumn{2}{|l|}{$\mathrm{ML}_{2}$} \\
\hline & 0 & $24 \mathrm{~h}$ & 0 & $24 \mathrm{~h}$ & 0 & $24 \mathrm{~h}$ \\
\hline$[\mathrm{LH}]^{+}$ & $\varnothing$ & $\varnothing$ & $(2 \%)$ & $\varnothing$ & $\sqrt{ }(6 \%)$ & $\sqrt{ }(11 \%)$ \\
\hline$[\mathrm{LAg}]^{+}$ & $\sqrt{ }(11 \%)$ & $\sqrt{ }(7 \%)$ & $\sqrt{ }(3 \%)$ & $\varnothing$ & $\sqrt{ }(1 \%)$ & $\sqrt{ }(4 \%)$ \\
\hline$\left[\mathrm{LAg}_{2}\left(\mathrm{NO}_{3}\right)\right]^{+}$ & $\sqrt{ }(100 \%)$ & $\sqrt{ }(100 \%)$ & $\sqrt{ }(34 \%)$ & $\sqrt{ }(37 \%)$ & $\sqrt{ }(18 \%)$ & $\sqrt{ }(26 \%)$ \\
\hline$\left[\mathrm{L}_{2} \mathrm{Ag}\right]^{+}$ & $\varnothing$ & $\varnothing$ & $\sqrt{ }(9 \%)$ & $\sqrt{ }(19 \%)$ & $\sqrt{ }(18 \%)$ & $\sqrt{ }(69 \%)$ \\
\hline$\left[\mathrm{LAg}_{3}\left(\mathrm{NO}_{3}\right)_{2}\right]^{+}$ & $\sqrt{ }(52 \%)$ & $\sqrt{ }(69 \%)$ & $\sqrt{ }(14 \%)$ & $\sqrt{ }(37 \%)$ & $\sqrt{ }(2 \%)$ & $\sqrt{ }(5 \%)$ \\
\hline$\left[\mathrm{L}_{2} \mathrm{Ag}_{2}\left(\mathrm{NO}_{3}\right)\right]^{+}$ & $\sqrt{ }(80 \%)$ & $\sqrt{ }(31 \%)$ & $\sqrt{ }(100 \%)$ & $\sqrt{ }(100 \%)$ & $\sqrt{ }(100 \%)$ & $\sqrt{ }(100 \%)$ \\
\hline$\left[\mathrm{L}_{2} \mathrm{Ag}_{3}\left(\mathrm{NO}_{3}\right)_{2}\right]^{+}$ & $\sqrt{ }(45 \%)$ & $\sqrt{ }(24 \%)$ & $\sqrt{ }(30 \%)$ & $\sqrt{ }(60 \%)$ & $\sqrt{ }(12 \%)$ & $\sqrt{ }(19 \%)$ \\
\hline$\left[\mathrm{L}_{2} \mathrm{Ag}_{4}\left(\mathrm{NO}_{3}\right)_{3}\right]^{+}$ & $\sqrt{ }(62 \%)$ & $\sqrt{ }(36 \%)$ & $\sqrt{ }(18 \%)$ & $\sqrt{ }(60 \%)$ & $\sqrt{ }(5 \%)$ & $\sqrt{ }(7 \%)$ \\
\hline$\left[\mathrm{L}_{3} \mathrm{Ag}_{3}\left(\mathrm{NO}_{3}\right)_{2}\right]^{+}$ & $\varnothing$ & $\varnothing$ & $\sqrt{ }(2 \%)$ & $\sqrt{ }(10 \%)$ & $\sqrt{ }(3 \%)$ & $\sqrt{ }(3 \%)$ \\
\hline$\left[\mathrm{L}^{3} \mathrm{Ag}_{4}\left(\mathrm{NO}_{3}\right)_{3}\right]^{+}$ & $\sqrt{ }(18 \%)$ & $\sqrt{ }(8 \%)$ & $\sqrt{ }(7 \%)$ & $\sqrt{ }(30 \%)$ & $\sqrt{ }(3 \%)$ & $\sqrt{ }(4 \%)$ \\
\hline
\end{tabular}




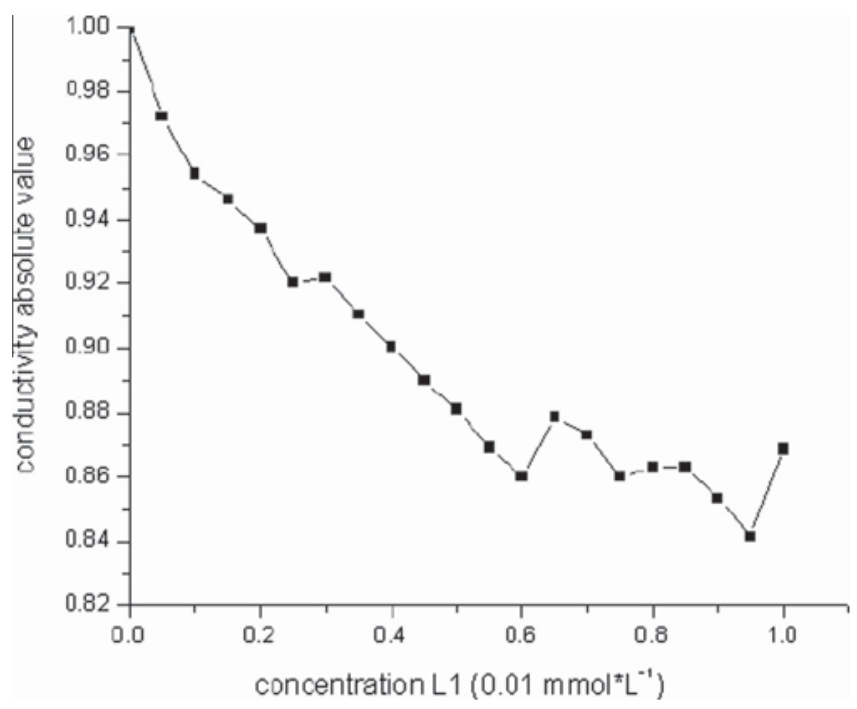

Fig. 6. Ionic conductivity of a THF-solution containing $\mathrm{AgNO}_{3}$ as a function of aliquots of Li1 added [11].

objects. The $\mathrm{PF}_{6}{ }^{-}$anion has furthermore the possibility to connect 1D-chains of the AgLi1 polymer into three dimensions, with the single chains criss-crossing each other. With the triflate anion, the chains are connected into porous, twice interconnected 3Dnetworks [7]. In contrast, the nitrate anion was only able to connect the 1D-chains into 2D-sheets, e.g. in the structure types 1 and 2 and when using all three $\mathrm{O}$-donors for bridging.

\subsection{Solution behavior}

How these compounds form from solution was investigated with electrospray mass spectrometry for different solvents. In acetonitrile, a strong competition between solvent and ligand occurs for coordination to the metal ion. Indeed, in solution, mostly complexes of $\mathrm{Ag}^{+}$with one or two molecules of acetonitrile were found. If $\mathrm{CH}_{2} \mathrm{Cl}_{2}$ however is added to the acetonitrile solution as of $10 \%$, the poor coordination ability of the solvent, hence the absence of donor atoms, leads to the formation of oligomers of the coordination polymer in solution. These oligomers were found to be mixtures of $[\mathrm{Li1H}]^{+} ; 379.1,[(\mathrm{Li} 1) \mathrm{Ag}]^{+} ; 549.9\left[(\mathrm{Li} 1) \mathrm{Ag}_{2}\left(\mathrm{NO}_{3}\right)\right]^{+} ; 650.9\left[(\mathrm{Li} 1)_{2} \mathrm{Ag}\right]^{+}$; $718.6\left[(\mathrm{Li} 1) \mathrm{Ag}_{3}\left(\mathrm{NO}_{3}\right)_{2}\right]^{+} ; 821.7\left[(\mathrm{Li} 1)_{2} \mathrm{Ag}_{2}\left(\mathrm{NO}_{3}\right)\right]^{+} ; 990\left[(\mathrm{Li} 1)_{2} \mathrm{Ag}_{3}\right.$ $\left.\left(\mathrm{NO}_{3}\right)_{2}\right]^{+} ; 1161.3\left[(\mathrm{Li} 1)_{2} \mathrm{Ag}_{4}\left(\mathrm{NO}_{3}\right)_{3}\right]^{+} ; 1262.2\left[(\mathrm{Li} 1)_{3} \mathrm{Ag}_{3}\left(\mathrm{NO}_{3}\right)_{2}\right]^{+}$; $1432.6\left[(\mathrm{Li} 1)_{3} \mathrm{Ag}_{4}\left(\mathrm{NO}_{3}\right)_{3}\right]^{+} \mathrm{m} / z$ [12]. Table 1 gives the relative percentage of each species as a function of metal to ligand ratio as well as time. It can be noticed that equilibria in solution lead to a variation of the respective complexes as a function of time as compared to immediately after mixing $(0 \mathrm{~h})$ and after $24 \mathrm{~h} \mathrm{[16-18] \text {. }}$

We could also show that adding better or less good solvents into the solution can influence the relative abundance of oligomers. Good solvents favor the generation of short oligomers in solution or even replace the ligand in its coordination to silver, while less good solvents show more presence of heavier oligomers.

Another experiment to elucidate the formation of coordination polymers in solution was done by using conductimetry [13]. To do so, the ligand Li1 and silver nitrate were dissolved in THF at a concentration of $0.01 \mathrm{mmol} / 1$ in order to remain under the solubility product of the coordination polymer to be formed and thus to keep all species in solution. The solution of Li1 was added in aliquots to the solution of $\mathrm{AgNO}_{3}$ and the ionic conductivity was measured after stabilization of the value (typically after one minute). It can be seen that with adding ligand to the silver salt solution, the ionic conductivity continuously decreases until a ratio of ca. $0.6: 1$ of Li1: $\mathrm{Ag}^{+}$is reached (Fig. 6). Then, the conductivity decreases more slowly until the ratio 1:1 is reached. This can be explained by the subsequent formation of complexes in solution, which become larger and contribute less to the conductivity.

\subsection{Crystal engineering aspects and conclusions}

From our previous results, it can be concluded that the structure prediction for silver coordination polymers in the case of Li1 remains difficult. It can be narrowed down to the formation of chains with alternating silver ions and ligand molecules as basic building blocks. In solution, the size and abundance of oligomers can be strongly tuned with the choice of the solvents, e.g. if they are themselves coordinating or not. Addition of non-coordinating solvents can push the equilibria also to longer oligomers and finally precipitation. The counter ions strongly influence the connectivity between the chains and are decisive for the formation of double chains, 2D and 3D structures. Their shape as such, as well as their number and orientation of donor atoms will play a crucial role for the construction of these networks. Finally, co-crystallizing solvents, often involved in $\mathrm{H}$-bonding to the anions or the polymer chains, can play an important role in the final structural outcome.

\subsection{Applications and outlooks}

Although difficult to predict, once the conditions found for obtaining a specific structural motif, a large scale production of the compounds can be envisaged. In our hands, we focus on several applications in the context of silver chemistry on one hand and the ligand Lin on the other. One is concerned with the generation of silver nanoparticles $[19,20]$, as we were able to show that our compounds possess good light stability in the cases where the silver ions are spaced from each other [15,7], or poor light stability if silver ions are arranged close to each other [7]. Another application deals with the antimicrobial properties of the silver ions, for which we were able to show that compounds e.g. of type 1 can be deposited layer by layer on the surface of metallic implant materials to show excellent antimicrobial properties [6,7,21-23]. Speaking of implant materials, one has also to consider the biocompatibility of the compounds, and we could show good biocompatibility in vitro for fibroblasts [6] and even in a mouse model in vivo [24]. The longer ligands become more flexible and water soluble [25] and feature not only chain compounds [11], but also metallacyclic species. The effect of the ligand length on the coordination modes towards silver ions has also been studied [14,26].

Such long, symmetrically as well as asymmetrically substituted ligands derived from oligoethylene glycols, of which Li1 is one member, can also be used to generate other metal ion complexes [27-31]. Among those, it might be interesting to highlight the heterobimetallic compounds which are low-temperature precursors leading to mixed metal oxide materials upon combustion [32]. The reader interested in more details on silver and its applications in medicine might refer to literature citations [33-36].

\section{Acknowledgements}

We thank the Swiss National Science Foundation and the University of Fribourg for continuous support and the Fribourg Center for Nanomaterials FriMat for co-funding of machines.

\section{References}

[1] For recent example: Li-Na Cui, Zhong-Feng Li, Qiong-Hua Jin, Xiu-Lan Xin, CunLin Zhang, Inorg. Chem. Commun. 20 (2012) 126.

[2] For recent example: Pilar Brandi-Blanco, Pablo J. Sanz Miguel, Bernhard Lippert, Eur. J. Inorg. Chem. (2012) 1122.

[3] For example: Ki-Min Park, Eunji Lee, Chul Soon Park, Shim Sung Lee, Inorg. Chem. 50 (2011) 12085. 
[4] For example: Irene Bassanetti, Francesco Mezzadri, Angiolina Comotti, Piero Sozzani, Marcello Gennari, Gianluca Calestani, Luciano Marchiò, J. Am. Chem. Soc. 134 (2012) 9142

[5] Yu Liu, Mei Pan, Qing-Yuan Yang, Lei Fu, Kang Li, Shi-Chao Wei, Cheng-Yong Su, Chem. Mater. 24 (2012) 1954.

[6] Priscilla S. Brunetto, Tünde Vig Slenters, Katharina M. Fromm, Materials 4 (2011) 355.

[7] T. Vig Slenters, J.L. Sagué, P.S. Brunetto, S. Zuber, A. Fleury, L. Mirolo, A.Y. Robin, M. Meuwly, O. Gordon, R. Landmann, A.U. Daniels, K.M. Fromm, Materials 3 (2010) 3407.

[8] For example: D.J. Hutchinson, S.A. Cameron, L.R. Hanton, S.C. Moratti, Inorg Chem. 51 (9) (2012) 5070.

[9] Adeline Y. Robin, Katharina M. Fromm, Helmut Goesmann, Gérald Bernardinelli, Cryst. Eng. Commun. 5 (2003) 405.

[10] K.M. Fromm, E.D. Gueneau, A.Y. Robin, W. Maudez, J. Sague, R. Bergougnant, Z. Anorg. Allg. Chem. 631 (2005) 1724.

[11] J.L. Sagué Doimeadios, A.Y. Robin, K.M. Fromm, Chem. Commun. 36 (2005) 4548.

[12] J.L. Sague, K.M. Fromm, Cryst. Growth Des. 6 (2006) 1566

[13] J. L. Sagué Doimeadios, Ph.D. Thesis, University Basel, Switzerland, 2006.

[14] I. Chevrier, J.L. Sagué, N. Khanna, K.M. Fromm, Dalton Trans. 42 (2013) 217.

[15] Adeline Y. Robin, Ph.D. Thesis, University of Basel, Switzerland, 2005.

[16] K.M. Fromm, A. Robin, M. Meuwly, H. Goesmann, G. Bernardinelli, Cryst. Eng. Commun. 6 (60) (2004) 336.

[17] A.Y. Robin, J.L. Sagué, K.M. Fromm, Cryst. Eng. Commun. 8 (2006) 403.

[18] A.Y. Robin, J.L. Sague Doimeadios, A. Neels, T. Vig Slenters, K.M. Fromm, Inorg. Chim. Acta 360 (2007) 212
[19] A. Mantion, A.G. Guex, A. Foelske, L. Mirolo, K.M. Fromm, M. Painsi, A. Taubert, Soft Matter 4 (2008) 606.

[20] K. Belser, T. Vig Slenters, C. Pfumbidzai, G. Upert, L. Mirolo, Angew. Chem., Int. Ed. 48 (2009) 3661.

[21] C.R. Arciola, N. Balaban, L. Baldassarri, K. Fromm, G.M. Hansch, U. Obst, E. Presterl, S. Stefani, J. Verran, L. Visai, Int. J. Artif. Organs 31 (2008) 858.

[22] P.S. Brunetto, K.M. Fromm, Chimia 62 (2008) 249.

23] Tünde Vig Slenters, Irmgard Hauser-Gerspach, Alma U. Daniels, Katharina M. Fromm, J. Mater. Chem. 18 (2008) 5359.

[24] O. Gordon, T. Vig, Antimicrob. Agents Chemother. 54 (2010) 4208.

[25] J.L. Sague, M. Meuwly, K.M. Fromm, CrystEngComm 10 (2008) 1542.

[26] F. Gschwind, K. M. Fromm, CrystEngComm 14 (2012) 4008

[27] A. Crochet, K.M. Fromm, Z. Anorg. Allg. Chem. 637 (2011) 2089.

[28] F. Gschwind, K.M. Fromm, Z. Anorg. Allg. Chem. 637 (2011) 1871.

29] W. Maudez, K. M. Fromm, Z. Anorg. Allg. Chem. 638 (2012) 1810.

[30] A. Crochet, K.M. Fromm, Z. Anorg. Allg. Chem. 636 (2010) 1484.

[31] F. Gschwind, A. Crochet, Chimia 64 (2010) 299.

[32] F. Gschwind, O. Sereda, K.M. Fromm, Inorg. Chem. 48 (2009) 10535

[33] K.M. Fromm, Nat. Chem. 3 (2011) 178.

[34] A.Y. Robin, K.M. Fromm, Coord. Chem. Rev. 250 (2006) 2127.

[35] S. Eckhardt, P.S. Brunetto, J. Gagnon, M. Priebe, B. Giese, K.M. Fromm, Chem Rev., accepted for publication.

[36] M.J. Hajipour, K.M. Fromm, A.A. Ashkarran, D.J. de Aberasturi, I.R. De Larramendi, T. Rojo, V. Serpooshan, W.J. Parak, M. Mahmoudi, Trends Biotechnol., http://dx.doi.org/10.1016/j.tibtech.2012.06.004. 